\title{
Diffuse X-ray emission from the Carina Nebula observed with Suzaku
}

\author{
K. Hamaguchi ${ }^{1,2}$, R. Petre ${ }^{1}$ and the Suzaku $\eta$ Carina team \\ ${ }^{1}$ NASA Goddard Space Flight Center, Greenbelt, MD 20771, USA \\ email: kenji@milkyway.gsfc.nasa.gov \\ ${ }^{2}$ Universities Space Research Association, 10211 Wincopin Circle, Suite 500, Columbia, \\ MD 21044-3432, USA
}

\begin{abstract}
The Carina Nebula possesses the brightest soft diffuse X-ray emission among the Galactic giant HII regions, but the origin has not been known yet. The XIS1 back-illuminated CCD camera onboard the Suzaku X-ray observatory has the best spectral resolution for extended soft sources so far, and is therefore capable of measuring these key emission lines in the soft diffuse plasma. Suzaku observed the Carina nebula on 2005 Aug. 29. The XIS1 spectra of the Carina nebula clearly showed spatial variations in emission line strengths. In the south, the spectrum showed strong L-shell lines of iron ions and K-shell lines of silicon ions, while in the north these lines were much weaker. Fitting the spectra with an absorbed thin-thermal plasma model with $k T \sim 0.2,0.6 \mathrm{keV}$ and $N_{\mathrm{H}} \sim 1-2 \times 10^{21} \mathrm{~cm}^{-2}$ showed that the silicon and iron abundance is about 2-3 times higher in the south than in the north. Because of its large size $(\sim 40 \mathrm{pc})$, the diffuse emission in the Carina nebula might have been produced by an old supernova, or a super shell produced by multiple supernovae.
\end{abstract}

Keywords. ISM: abundances, supernova remnants, X-rays: ISM
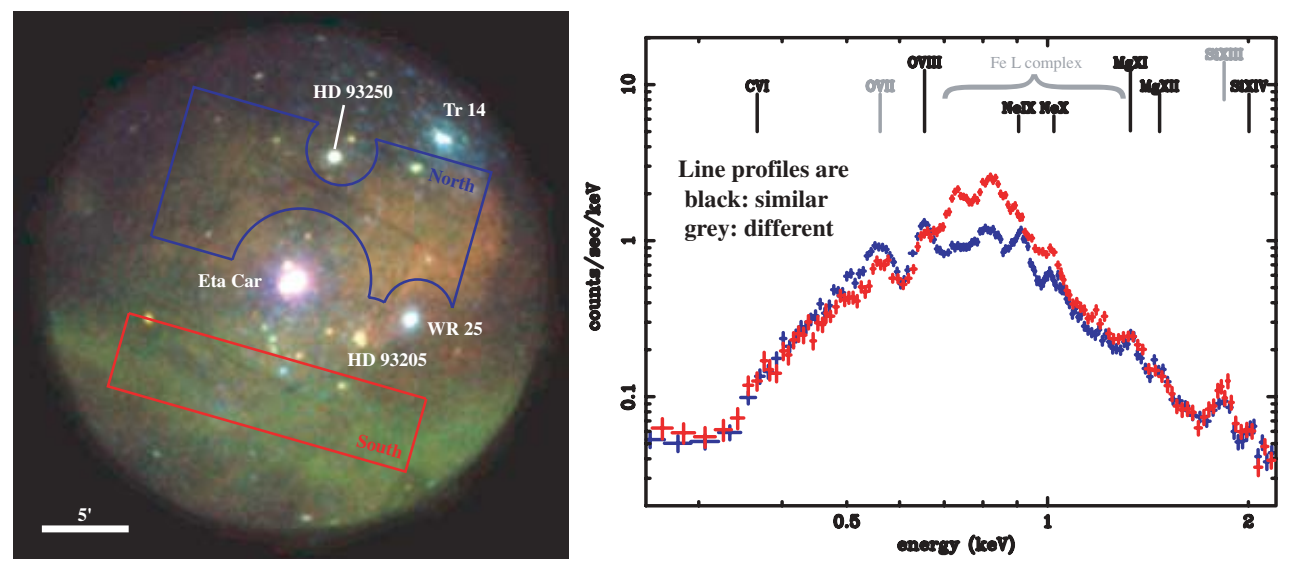

Figure 1. Left: XMM-Newton image with the source regions defined. Right: Suzaku normalized spectra of the north (lower curve) and south. See details for Hamaguchi et al. (2006).

\section{Acknowledgements}

K. H. is financially supported by the US Chandra grant No. GO3-4008A.

\section{Reference}

Hamaguchi et al. 2006, PASJ submitted 